ESAIM: PROCEEDINGS, December 2013, Vol. 43, p. 213-224

S. Descombes, B. Dussoubs, S. Faure, L. Gouarin, V. Louvet, M. Massot, V. Miele, Editors

\title{
A PARALLEL IMPLEMENTATION OF THE MORTAR ELEMENT METHOD IN 2D AND 3D
}

\author{
A. Samake ${ }^{1}$, S. Bertoluzza ${ }^{2}$, M. Pennacchio ${ }^{3}$, C. Prud'homme ${ }^{4}$ et C. Zaza ${ }^{5}$
}

\begin{abstract}
We present here the generic parallel computational framework in $\mathrm{C}++$ called FeEL ++ for the mortar finite element method with the arbitrary number of subdomain partitions in $2 \mathrm{D}$ and $3 \mathrm{D}$. An iterative method with block-diagonal preconditioners is used for solving the algebraic saddle-point problem arising from the finite element discretization. Finally we present a scalability study and the numerical results obtained using FEEL ++ library.
\end{abstract}

Keywords: Domain decomposition and mortar method and parallel computing

\section{INTRODUCTION}

Domain decomposition methods are becoming increasingly popular as a tool to solve problems arising in many different applications. The possibility of easily coupling different discretizations and/or different numerical methods in different subdomains without the need of imposing strong matching conditions is the main feature of the nonconforming version of such methods and adds a further advantage.

In this paper we consider an algorithm presented in [2] for solving the linear system arising from the mortar element method (initially proposed by C. Bernardi, Y. Maday and A. Patera in [8]) as well as another variant with Lagrange multipliers proposed by F. Ben Belgacem and Y. Maday in [6]. The main feature of the Mortar method is that the interface continuity conditions of the subdomains is taken into account in weak form by asking the jump of the finite element solution on the interface to be $L_{2}$-orthogonal to a well chosen Lagrange multiplier space. The approximation properties of such a method are optimal in the sense that the error is bounded by the sum of the subdomain approximation errors.

In order to make such techniques more competitive for real life applications, one has to deal with the problem of efficient implementation. As it happens with all domain decomposition methods (both conforming and nonconforming) the efficient implementation relies on parallelizing the solution process by assigning each subdomain to a processor and employing the preferred iterative scheme.

The paper is organized as follows. In section 2 we recall the mortar finite element method with Lagrange multipliers. In section 3 the computational framework is described and some remarks on the parallel implementation aspects are given. Finally, the numerical results showing strong and weak scaling on large architectures in $3 \mathrm{D}$ are presented in section 4 and we give brief conclusions in section 5.

\footnotetext{
${ }^{1}$ Université Joseph Fourier Grenoble 1, / CNRS, Laboratoire Jean Kuntzmann / UMR 5224. Grenoble, F-38041, France

(C) EDP Sciences, SMAI 2013 


\section{The Mortar Method}

Let $\Omega$ be bounded domain of $\mathbb{R}^{d}, d=2,3$. We denote $\partial \Omega$ its boundary and we assume that $\Omega$ is a union of $L$ subdomains $\Omega_{k}$ :

$$
\Omega=\bigcup_{k=1}^{L} \Omega_{k} .
$$

We assume that the domain decomposition is geometrically conforming. It means that if $\gamma_{k l}=\bar{\Omega}_{k} \cap \bar{\Omega}_{l}(k \neq l)$ and $\gamma_{k l} \neq \emptyset$, then $\gamma_{k l}$ must either be a common vertex of $\Omega_{k}$ and $\Omega_{l}$, or a common edge, or a common face. We define $\Gamma_{k l}=\gamma_{k l}$ as the interface between $\Omega_{k}$ and $\Omega_{l}$. We note that $\Gamma_{k l}=\Gamma_{l k}$.

We consider the Dirichlet boundary value problem (2): find $u$ satisfying

$$
\begin{aligned}
-\Delta u=f & \text { in } \quad \Omega, \\
u=g & \text { on } \quad \partial \Omega,
\end{aligned}
$$

where $f \in L^{2}(\Omega)$ and $g \in H^{1 / 2}(\partial \Omega)$ are given functions. The usual variational formulation of (2) reads as follows

Problème 2.1. Find $u \in H_{g}^{1}(\Omega)$ such that

$$
\int_{\Omega} \nabla u \cdot \nabla v d x=\int_{\Omega} f v d x \quad \forall v \in H_{0}^{1}(\Omega)
$$

where $H_{g}^{1}$ denotes the space $H_{g}^{1}=\left\{u \in H^{1}(\Omega), u=g\right.$ on $\left.\partial \Omega\right\}$.

Let us denote by $H^{1 / 2}\left(\Gamma_{k l}\right)$ the trace space of one of the spaces $H^{1}\left(\Omega_{k}\right)$ or $H^{1}\left(\Omega_{l}\right)$ on the interface $\Gamma_{k l}$. We define two product spaces:

$$
V=\prod_{k=1}^{L} H^{1}\left(\Omega_{k}\right), \quad \Lambda=\prod_{k=1}^{L} \prod_{\substack{0 \leq l<k \\\left|\Gamma_{k l}\right| \neq 0}}\left(H^{1 / 2}\left(\Gamma_{k l}\right)\right)^{\prime} .
$$

The space $\Lambda$ will be a trial space for the weak continuity conditions on the interfaces. We introduce the bilinear forms $a: V \times V \rightarrow \mathbb{R}, b: V \times \Lambda \rightarrow \mathbb{R}$ and the linear functional $f: V \rightarrow \mathbb{R}$ :

$$
\begin{aligned}
a(\mathbf{u}, \mathbf{v}) & =\sum_{k=1}^{L} a_{k}(\mathbf{u}, \mathbf{v}), \quad a_{k}(\mathbf{u}, \mathbf{v})=\int_{\Omega_{k}} \nabla u_{k} \cdot \nabla v_{k} d x, \\
b(\lambda, \mathbf{v}) & =\sum_{k=1}^{L} \sum_{\substack{l=0 \\
\left|\Gamma_{l k}\right| \neq 0}}^{L} b_{k l}(\lambda, \mathbf{v}), \quad b_{k l}(\lambda, v)=\left.\left\langle\lambda_{k l}, v_{k}\right\rangle\right|_{\Gamma_{k l}}, \\
f(\mathbf{v}) & =\sum_{k=1}^{L} \int_{\Omega_{k}} f v_{k} d x,
\end{aligned}
$$

where $\lambda_{k l}=-\lambda_{l k}$ and $\left.\langle\cdot, \cdot\rangle\right|_{\Gamma_{k l}}$ stands for the duality product between $\left(H^{1 / 2}\left(\Gamma_{k l}\right)\right)^{\prime}$ and $H^{1 / 2}\left(\Gamma_{k l}\right)$. The bilinear form $a_{k}(\cdot, \cdot)$ corresponds to the Dirichlet problem in the subdomain $\Omega_{k}$ for the operator $-\Delta$.

The approach that we consider here is the mortar formulation with Lagrange multipliers presented in [2] that reads as follows: 
Problème 2.2. Find $(\mathbf{u}, \lambda) \in V \times \Lambda$ such that

$$
\begin{aligned}
a(\mathbf{u}, \mathbf{v})+b(\lambda, \mathbf{v}) & =f(\mathbf{v}) \\
b(\mu, \mathbf{u}) & =0 \\
\forall(\mathbf{v}, \mu) & \in V \times \Lambda
\end{aligned}
$$

If $\tilde{\mathbf{u}}$ and $\tilde{\lambda}$ denote the vectors of the components of $\mathbf{u}_{h}$ and $\lambda_{h}$, finite element approximations to $\mathbf{u}$ and $\lambda$, the discrete problem associated to the problem (2.2) is equivalent to a saddle-point system of the following form:

where

$$
\mathcal{A}\left(\begin{array}{c}
\tilde{\mathbf{u}} \\
\tilde{\lambda}
\end{array}\right)=\left(\begin{array}{c}
f \\
0
\end{array}\right), \quad \mathcal{A}=\left(\begin{array}{cc}
A & B^{T} \\
B & 0
\end{array}\right)
$$

$$
A=\left(\begin{array}{ccc}
A_{1} & & 0 \\
& \ddots & \\
0 & & A_{L}
\end{array}\right), \quad B^{T}=\left(\begin{array}{c}
B_{1}^{T} \\
\vdots \\
B_{L}^{T}
\end{array}\right)
$$

and $A_{k}$ corresponds to the stiffness matrix in the subdomain $\Omega_{k}$ and $B_{k}$ denotes the matrix associated to the discrete form of the mortar weak continuity constraint in the subdomain $\Omega_{k}$.

Remarque 2.3. Moreover, there are other mortar formulations namely master-slave constraint formulation in which the weak continuity constraint is directly taken into account in the approximations space called constraint space.

\section{Computational Framework}

\subsection{Krylov Iterative solvers}

We want to solve the saddle-point problem (6) using an iterative Krylov subspace method in parallel. Finding a good preconditioner for such problems is a delicate issue as the matrix $\mathcal{A}$ is indefinite and any preconditioning matrix $\mathcal{P}$ acting on the jump matrices $B_{k l}$ would involve communications.

A survey on block diagonal and block triangular preconditioners for this type of saddle-point problem (6) can be found in $[7,12]$.

The matrix $\mathcal{A}$ arising in saddle-point problems is known to be spectrally equivalent to the block diagonal matrix:

$$
\mathcal{P}=\left(\begin{array}{cc}
A & 0 \\
0 & -S
\end{array}\right)
$$

where $S$ is the Schur complement $-B A^{-1} B^{T}$ (see for instance [15]). While not being an approximate inverse of $\mathcal{A}$, the matrix $\mathcal{P}$ is an ideal preconditioner. Indeed it can be shown that $P(X)=X(X-1)\left(X^{2}-X-1\right)$ is an annihilating polynomial of the matrix $\mathcal{T}=\mathcal{P}^{-1} \mathcal{A}$. Therefore, assuming $\mathcal{T}$ non singular, the matrix $\mathcal{T}$ has only three eigenvalues $\{1,(1 \pm \sqrt{5}) / 2\}$. Thus an iterative solver using the Krylov subspaces constructed with $\mathcal{T}$ would converge within three iterations. In practice, computing the inverse of the exact preconditioner $\mathcal{P}$ is too expensive. Instead, one would rather look for an inexact inverse $\hat{\mathcal{P}}^{-1}$. When applying the preconditioner, the inexact inverse $\hat{\mathcal{P}}^{-1}$ would be determined following an iterative procedure for solving the linear system $\mathcal{P} \mathbf{x}=\mathbf{y}$. This requires a class of iterative methods qualified as flexible inner-outer preconditioned solvers [18] or inexact inner-outer preconditioned solvers [13].

The outer iterations for solving the main problem would involve inner iterations for computing an inexact and non-constant preconditioner. Finding the relevant convergence parameters to this inner iterative procedure is a critical issue. On the one hand, $\hat{\mathcal{P}}^{-1}$ has to be computed in few iterations: the total number of iterations 
including the inner iterations should be less than without preconditioner. On the other hand for ensuring the stability of the outer iterations, it would be preferable to solve the inner iterations with as much accuracy as possible in order to keep an almost constant preconditioner. We refer the reader to [11] and references therein for theoretical results and experimental assessment with respect to the influence of the perturbation to the preconditioner. In this context, the choice a good preconditioner for solving the inner iterations can have a significant impact on the convergence of the outer iterations.

In this work the outer iterations will be carried out with a flexible preconditioned Biconjugate Gradient Stabilized Method (FBICGSTAB) (see for instance algorithm (7.7) in [19]) and the flexible preconditioned Generalized Minimal Residual Method (FGMRES (m)) with restart (see [20]): 


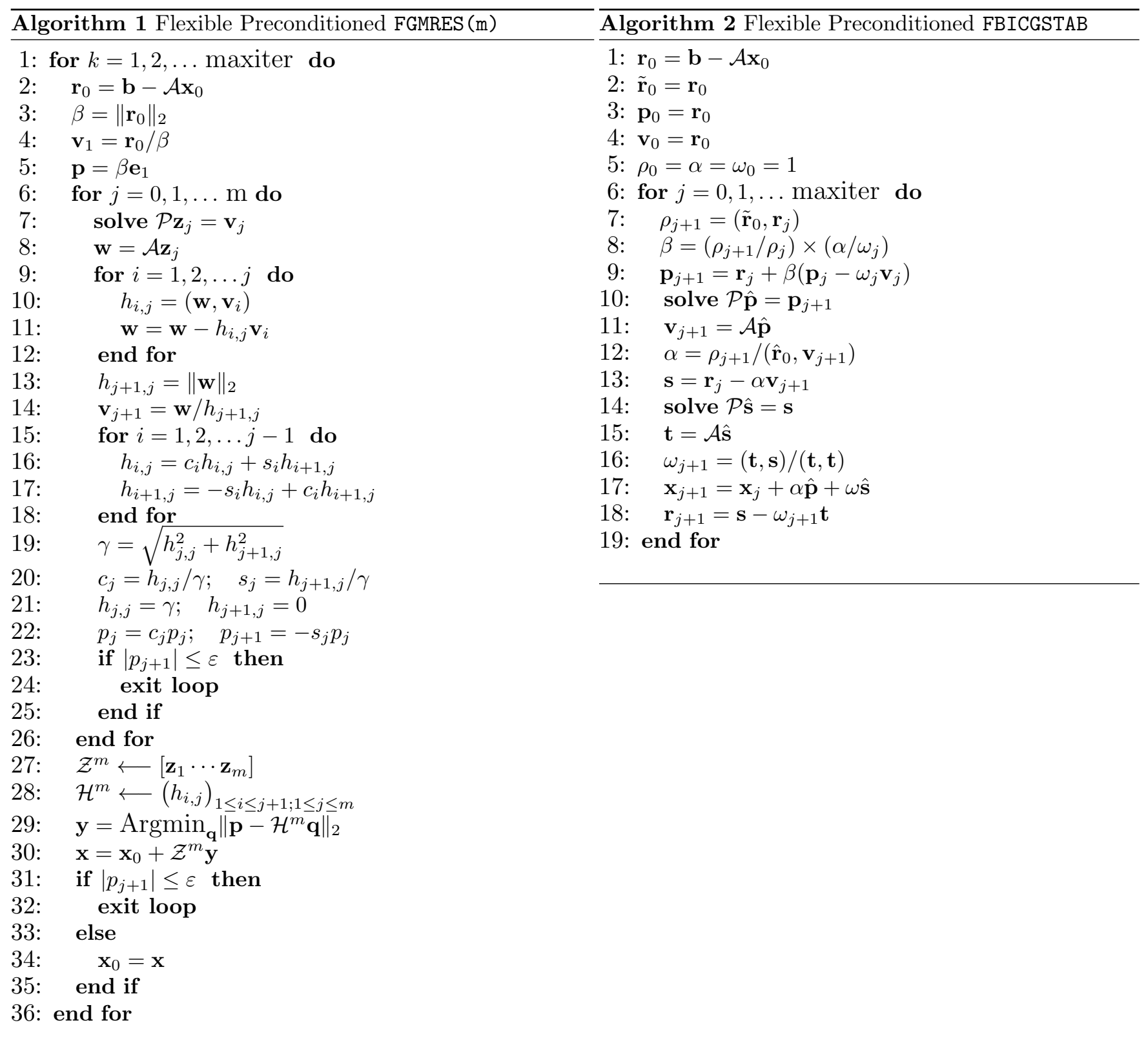

Regarding the preconditioning we will focus on two approximations of $\mathcal{P}$ :

$$
\mathcal{P}_{I}=\left(\begin{array}{cc}
A & 0 \\
0 & I
\end{array}\right) \quad \text { and } \quad \mathcal{P}_{S}=\left(\begin{array}{cc}
A & 0 \\
0 & -\hat{S}
\end{array}\right)
$$

In the first preconditioner the exact inverse of $\mathcal{P}_{I}$ is computed at each iteration using the $(I) L U$ factorization of the block diagonal matrix $A$. This preconditioner only acts on the diagonal blocks $A_{k}$. As a result, solving the linear system $\mathcal{P}_{I} \mathbf{x}=\mathbf{y}$ does not involve any communication between the subdomains. However, since $\mathcal{P}_{I}$ does not act on the jump matrices $B_{k l}$, it is very likely to become less effective as the number of subdomains increases. 
In the second preconditioner the exact inverse of the block diagonal matrix $A$ is also computed so that the exact Schur complement $S=-B A^{-1} B^{T}$ is readily available. Instead of taking $S$ we choose an approximation $\hat{S}$ such that $\hat{\mathbf{x}}=\hat{S}^{-1} \mathbf{y}$ is an approximate solution to the linear system $S \mathbf{x}=\mathbf{y}$ following an iterative procedure. This inner procedure is also carried out with a BICGSTAB algorithm preconditioned with the diagonal of $S$ (Jacobi preconditioner $\mathcal{M}_{J}$ ) or with $\mathcal{M}_{S}^{-1}=B A B^{T}$.

Remarque 3.1. The Krylov methods FBICGSTAB and FGMRES (m) are both adapted to our saddle-point problem, but the only major difference between these methods is that FBICGSTAB presents sometimes breakdowns unlike FGMRES $(m)$.

\subsection{Parallel implementation}

The parallel implementation is designed using the message passing interface(MPI) library. The objective of the parallel implementation is to minimize the amount of communications with respect to the parallel operations involved in the linear solver, namely matrix-vector products and dot products. One of interests of this mortar parallel implementation is that there's no communication at cross-points(in 2D and 3D) and cross-edges(in 3D), which reduces considerably communications between subdomains.

Assuming a constant number of internal dofs in each subdomain, it is rather straightforward to bind a subdomain to each process. Each process would own its subdomain mesh $\mathcal{T}_{h_{k}}$, functional space $X_{h_{k}}$, stiffness matrix $A_{k}$ and unknown $u_{k}$. Regarding the mortars, the choice is less obvious. In order to decrease the amount of communications in the matrix-vector products, we have used technique developed in [2] which consists in duplicating the data at the interfaces between subdomains. If $\Gamma_{k l}$ is such an interface, then the Lagrange multiplier vector $\lambda_{k l}$ and its associated trace mesh $\mathcal{T}_{h_{k}, l}$ and trace space $\mathcal{M}_{h_{k}, l}$ are stored in both the processors dealing $\Omega_{k}$ and $\Omega_{l}$. Although the data storage is increased a little bit, the communications will be reduced significantly.

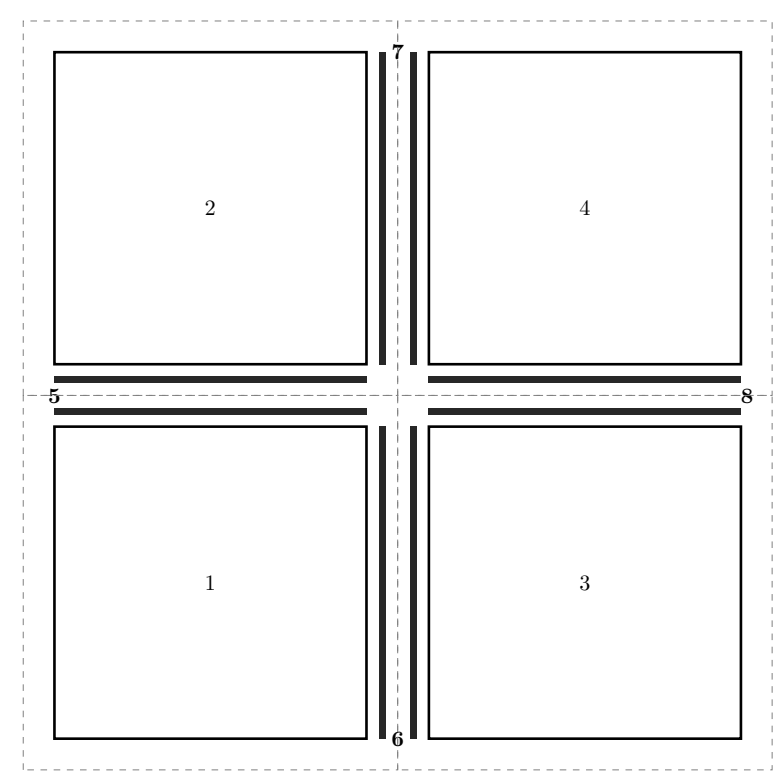

1.1. One subdomain per cluster

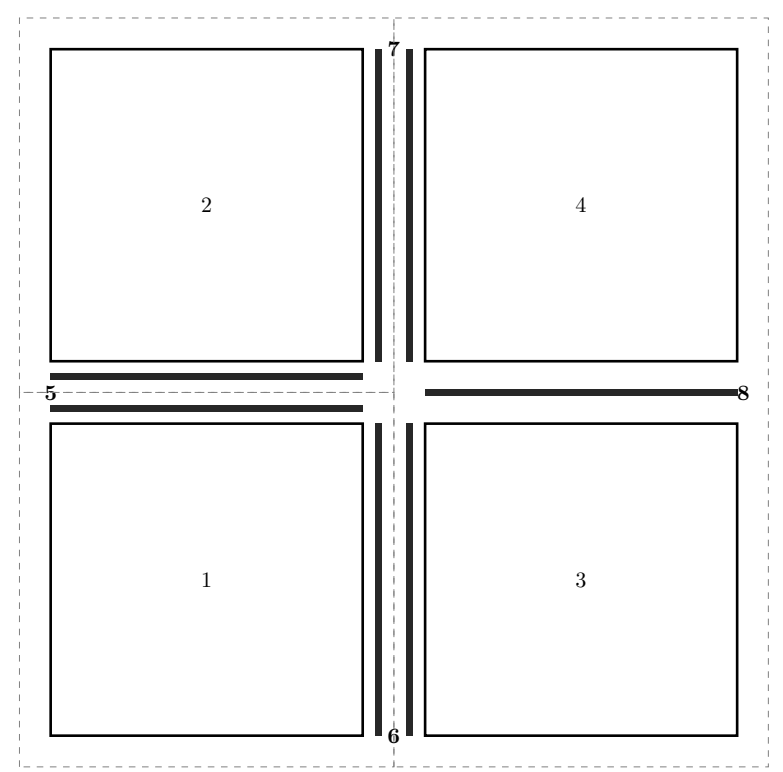

1.2. Subdomains 3 and 4 on the same cluster

Figure 1. Domain decompositions

As an example, consider the splitting of the unit square into four little squares, as in FigURE 1., where the dash rectangles denote clusters and the bold segments correspond to the mortar interfaces. Note, that when 
neighboring subdomains belong to different clusters, there are two copies of the mortar interface variables stored in different clusters. Consider the interfaces as shown in the picture. The matrix $\mathcal{A}$ has the following form:

$$
\mathcal{A}=\left(\begin{array}{cccc|cccc}
A_{1} & & & & B_{15}^{T} & B_{16}^{T} & \mathbf{0} & \mathbf{0} \\
& A_{2} & & & B_{25}^{T} & \mathbf{0} & B_{27}^{T} & \mathbf{0} \\
& & A_{3} & & \mathbf{0} & B_{36}^{T} & \mathbf{0} & B_{38}^{T} \\
& & & A_{4} & \mathbf{0} & \mathbf{0} & B_{47}^{T} & B_{48}^{T} \\
\hline B_{15} & B_{25} & \mathbf{0} & \mathbf{0} & & & & \\
B_{16} & \mathbf{0} & B_{36} & \mathbf{0} & & & & \\
\mathbf{0} & B_{27} & \mathbf{0} & B_{38} & & & & \\
\mathbf{0} & \mathbf{0} & B_{47} & B_{48} & & & &
\end{array}\right)
$$

Let us consider the matrix-vector multiplication procedure with the matrix $\mathcal{A}$ and the vector $(\tilde{u}, \tilde{\lambda})$, where $\tilde{u}$ and $\tilde{\lambda}$ have the following component-wise representation, according to the decomposition and the enumeration in Figure 1.1.: $\tilde{\mathbf{u}}=\left(u_{1}^{T}, u_{2}^{T}, u_{3}^{T}, u_{4}^{T}\right)^{T}$ and $\tilde{\lambda}=\left(\lambda_{5}^{T}, \lambda_{6}^{T}, \lambda_{7}^{T}, \lambda_{8}^{T}\right)^{T}$. The resulting vector $(\tilde{\mathbf{v}}, \tilde{\mu})=\mathcal{A} \cdot(\tilde{\mathbf{u}}, \tilde{\lambda}) \operatorname{can}$ be computed as

$$
\left(\begin{array}{c}
v_{1}^{(1)} \\
v_{2}^{(2)} \\
v_{3}^{(3)} \\
v_{4}^{(4)} \\
\hline \mu_{5}^{(1,2)} \\
\mu_{6}^{(1,3)} \\
\mu_{7}^{(2,3)} \\
\mu_{8}^{(3,4)}
\end{array}\right)=\left(\begin{array}{l}
A_{1} u_{1}^{(1)}+B_{15}^{T} \lambda_{5}^{(1)}+B_{16}^{T} \lambda_{6}^{(1)} \\
A_{2} u_{2}^{(2)}+B_{25}^{T} \lambda_{5}^{(2)}+B_{27}^{T} \lambda_{7}^{(2)} \\
A_{3} u_{3}^{(3)}+B_{36}^{T} \lambda_{6}^{(3)}+B_{38}^{T} \lambda_{8}^{(3)} \\
A_{4} u_{4}^{(4)}+B_{47}^{T} \lambda_{7}^{(4)}+B_{48}^{T} \lambda_{8}^{(4)} \\
B_{15} u_{1}^{(1)}+B_{25} u_{2}^{(2)} \\
B_{16} u_{1}^{(1)}+B_{36} u_{3}^{(3)} \\
B_{27} u_{2}^{(2)}+B_{47} u_{4}^{(4)} \\
B_{38} u_{3}^{(3)}+B_{48} u_{4}^{(4)}
\end{array}\right)
$$

where the upper indices denote the cluster(the processor), in which this variable is stored. Two upper indices mean that this variable is stored in both processors. Note that $\lambda_{i}^{(k)} \equiv \lambda_{i}^{(l)}$ and so far we need communications only when computing $\mu_{i}$. For example

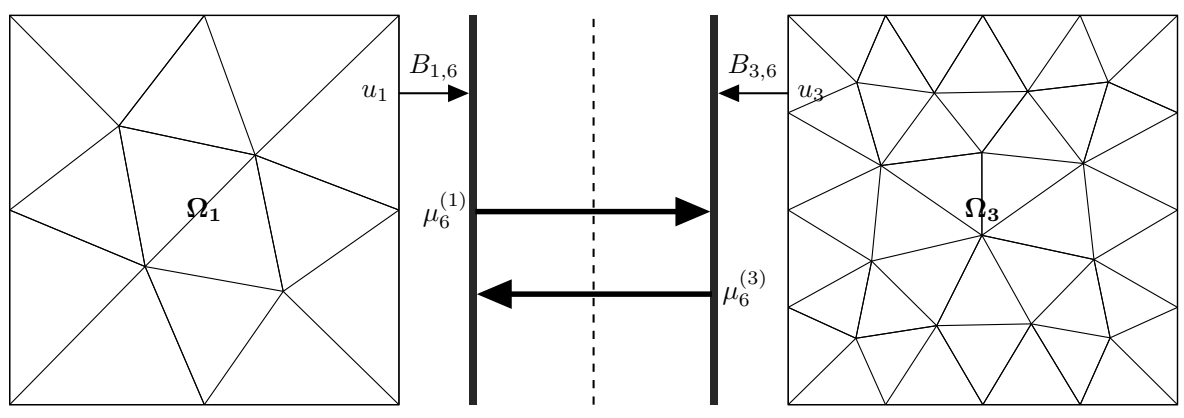

Figure 2. Communications for jump matrix multiplication

$$
\mu_{6}=\mu_{6}^{(1)}+\mu_{6}^{(3)}, \quad \mu_{6}^{(1)}=B_{16} u_{1} \text { and } \mu_{6}^{(3)}=B_{36} u_{3}
$$

We see that $\mu_{6}^{(1)}$ and $\mu_{6}^{(3)}$ are computed in parallel, and then should be interchanged and summed(see the representations in FiguRE 3. and more explicitly in FiguRE 2.) . 


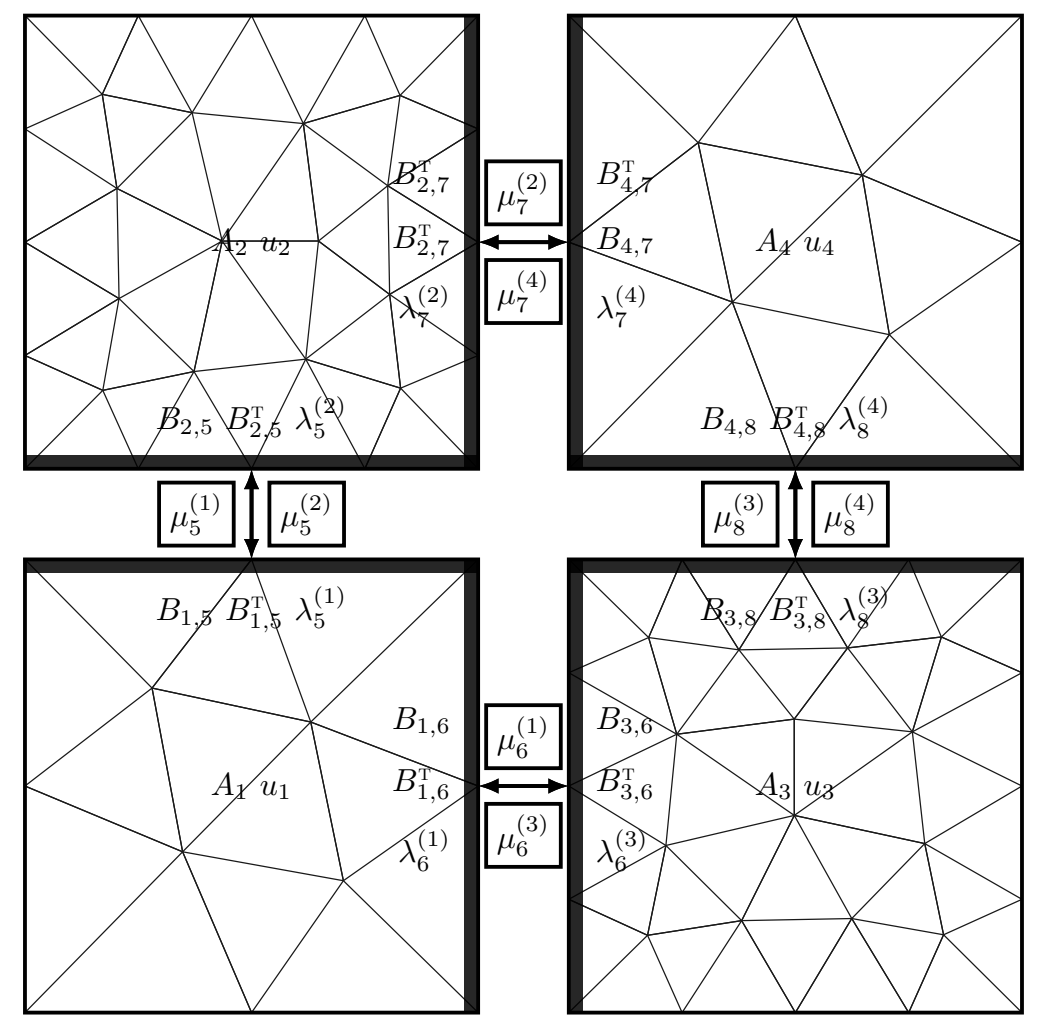

Figure 3. Communications for parallel matrix-vector multiplication

For the FEEL $++[16,17]$ implementation the communications are handled explicitly by the user and we use PETSC $[3-5,14]$ sequentially even though the code is parallel using MPI communicators. This technique requires explicitly sending and receiving complex data structures such as mesh data structures, PETSC vectors and elements of functions space(traces) using Boost.MPI and Boost. Serialization [1].

\section{Numerical Results}

We present in this section the numerical results of the parallel implementation of the mortar element method described in the section (3) using FeEL ++ and Boost.MPI libraries. We consider here the problem (2) in 3D with $g=\sin (\pi x) \cos (\pi y) \cos (\pi z)$ the exact solution and $f=-\Delta g=3 \pi^{2} g$ the corresponding right hand side. The problem is solved in the parallelepiped $\Omega=\left[0, L_{x}\right] \times\left[0, L_{y}\right] \times\left[0, L_{z}\right], L_{x}, L_{y}, L_{z}>0$. The following numerical results are obtained using $P_{2}$ finite element approximations in each subdomain $\Omega_{k}$ with $h_{\Omega_{k}}=0.1$ in the strong scaling study(see subsection 4.1) and $h_{\Omega_{k}}=0.075$ in the weak scaling one(see subsection 4.2), $k=1, \cdots, L$. The stopping criterion is such that the residual norm for the Krylov solver is less than $\varepsilon=10^{-7}$.

The simulations have been performed at Leibniz Supercomputing Centre (LRZ) on SuperMUC. SuperMUC is the Tier-0 supercomputer with 155.656 processor cores in 9400 compute nodes which provides resource to PRACE via the German Gauss Centre. The system is an IBM System x iDataPlex based on Intel Sandy Bride EP processors. SuperMUC has a peak performance of $3.2 \mathrm{PFLOP} / \mathrm{s}$ consisting of 18 islands, each combining 512 compute nodes with 16 physical cores and $32 \mathrm{~GB}$ per node. The nodes are connected by a non-blocking fat tree, based on Infiniband FDR10.

In the context of High Performance Computing(HPC), there are two common notions of scalability to evaluate the efficiency of the parallel computing. 
The first is the strong scaling, which is defined as how the solution time varies with the number of cores for a fixed total problem size. The goal is to minimize time to solution for a given problem by keeping the problem size fixed and increasing the number of cores.

The second is the weak scaling, which is defined as how the solution time varies with the number of cores for a fixed problem size per core. The goal is to solve the larger problems by keeping the work per core fixed and increasing the number of cores.

\subsection{Strong Scaling}

We present here the strong scaling results corresponding to the partition of the global domain $\Omega$ into $L_{x} \times$ $L_{y} \times L_{z}$ subdomains (1 subdomain per core) with the fixed lengths $L_{x}=L_{y}=L_{z}=1$. We plot in Figure 4.1. the absolute solve time and assembly time and total time versus the number of cores in loglog axis and in FiguRE 4.2. the speedup and ideal speedup versus number of cores. The total number of degrees of freedom is approximately equal to 500.000 and all the the measured timings are expressed in seconds.

We define the speedup by the following formula: $S_{p}=T_{r} / T_{p}$ where $p$ is the number of cores and $T_{r}$ the execution time of the parallel algorithm with $r \operatorname{cores}\left(r=4\right.$ is our reference number of cores) and $T_{p}$ the execution time of the parallel algorithm with $p$ cores $(r<p)$. Analogously we define by $S S_{p}$ the corresponding speedup for the solver time and by $A S_{p}$ for assembly.

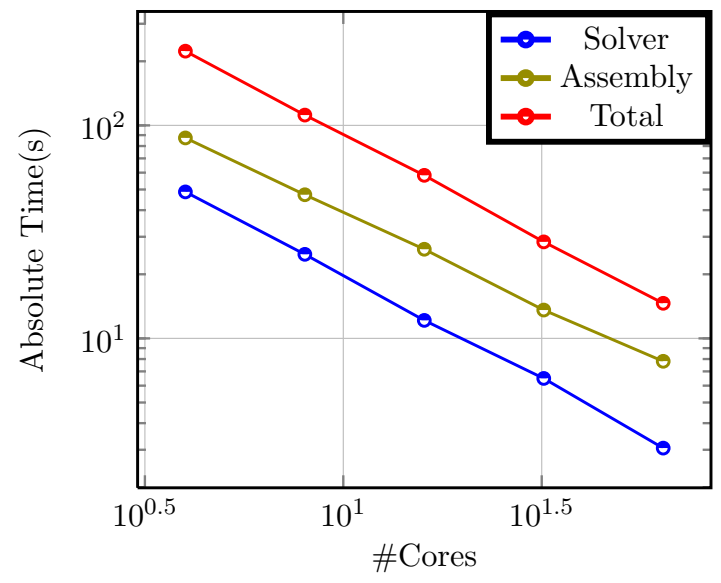

4.1. Absolute Solve and Total time versus \#Cores

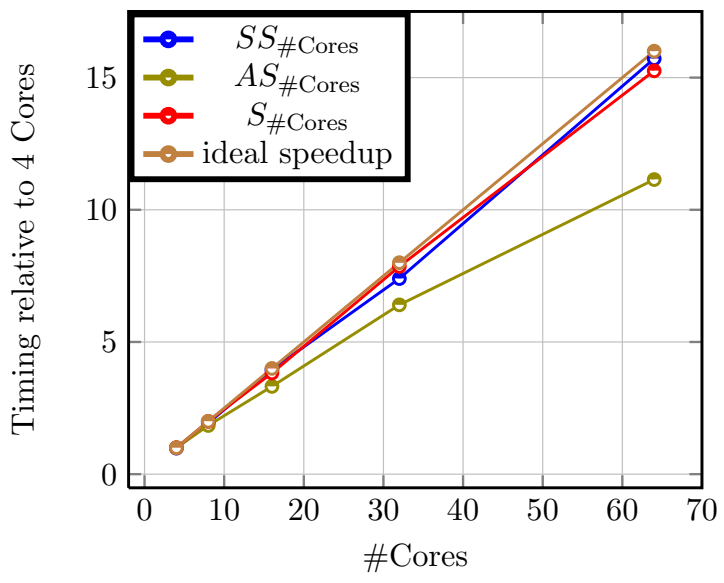

4.2. Speedup versus \#Cores

FIgURE 4. Strong scaling

Remarque 4.1. We observe in Figure 4.2. that the speedup related to the total computational time is very over the ideal speedup. This is due to the fact that the functions spaces construction and matrix factorization timings decrease significantly in the strong scaling, as well as the communications between subdomains which are few in only 24 cores. We expect to find the normal behavior on the large scale architectures on which we can take many subdomains with more consistent problem size.

\subsection{Weak Scaling}

We present here the the weak scaling results corresponding to the partition of the global domain $\Omega$ into $L_{x} \times L_{y} \times L_{z}$ subdomains (1 subdomain per processor) with $L_{x} \times L_{y} \times L_{z}=$ \#Cores. We plot in FiguRE 5.1. the absolute solve time and total time versus the number of cores in loglog axis and in Figure 5.2. the efficiency relative to four cores versus number of cores. We denote by $E_{p}$ the efficiency relative to four cores for the total time on $p$ cores and $E S_{p}$ the efficiency relative to four cores for the solver time on $p$ cores. The 
number of degrees of freedom is approximately equal to 30.000 per subdomain and all the measured timing are expressed in seconds.

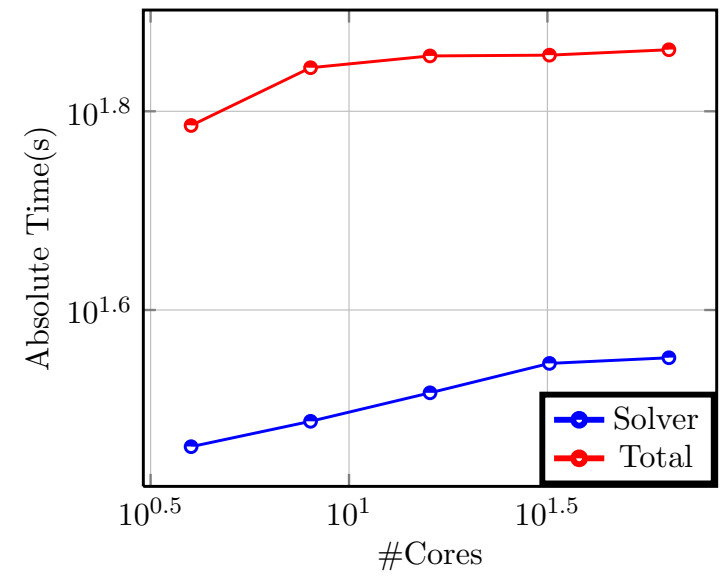

5.1. Absolute Solve and Total time versus \#Cores

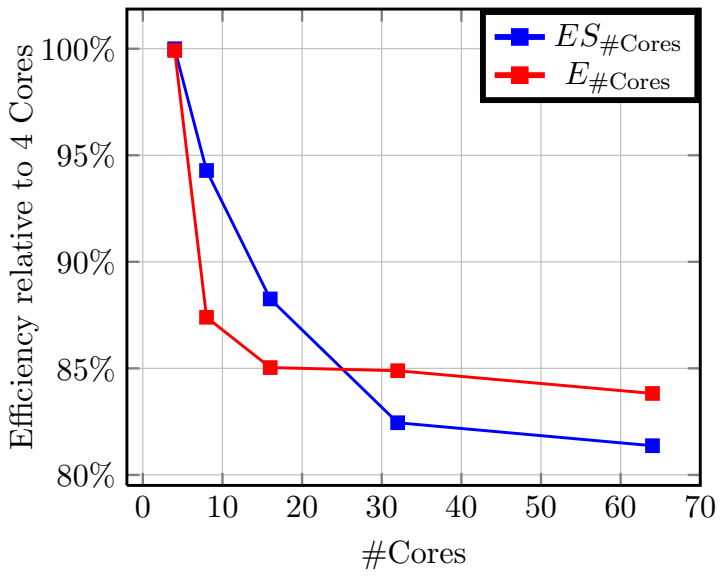

5.2. Efficiency versus \#Cores

FiguRE 5. Weak scaling

Remarque 4.2. The plots in FigurE 5.1. clearly show that the absolute solve and total time does not increase significantly when the number of cores and the problem size increase by keeping the problem size per core. This confirms the results expected for the weak scaling study.

\subsection{Convergence Results}

We summarize in the following Tables TABLE 1. and TABLE 2. the behavior of $L_{2}$ and $H^{1}$ errors of the numerical solution relative to the analytical solution of our problem using the mortar finite formulation described in the section 2 according to the maximum mesh size $h \in\{0.2,0.1,0.05,0.025\}$ and the Lagrange polynomial orders $P_{N}, N \in\{1,2,3\}$. The tests are performed in the nonconforming case where the characteristic mesh size in the subdomain $\Omega_{k}$ is $h_{\Omega_{k}}=h+\delta_{k}, k=1, \cdots, L$, with $\delta_{k}=0.001$ the small perturbation. All the tests are achieved with $2,4,8$ and 16 number of subdomains. We denote by $u$ the exact solution of our problem and $u_{h}^{N}$ the discrete solution obtained by using the characteristic size equal to $h$ and the piecewise polynomials of degree less than or equal to $N$. We denote $\|\cdot\|_{0}$ the $L^{2}$-norm and $\|\cdot\|_{1}$ the $H^{1}$-norm. We plot in FigurE 6.1 . and FiguRE 6.2. the $L^{2}$ and $H^{1}$ norms of the error relative to the exact solution versus the characteristic mesh sizes in loglog axis.

TABLE 1. $L^{2}$ Convergence results

\begin{tabular}{lccc}
\hline $\mathrm{h}$ & $\left\|u-u_{h}^{1}\right\|_{0}$ & $\left\|u-u_{h}^{2}\right\|_{0}$ & $\left\|u-u_{h}^{3}\right\|_{0}$ \\
\hline 0.2 & $2.80 \cdot 10^{-2}$ & $2.67 \cdot 10^{-3}$ & $2.16 \cdot 10^{-4}$ \\
0.1 & $6.69 \cdot 10^{-3}$ & $2.83 \cdot 10^{-4}$ & $9.58 \cdot 10^{-6}$ \\
0.05 & $1.66 \cdot 10^{-3}$ & $3.24 \cdot 10^{0}$ & $5.29 \cdot 10^{-7}$ \\
0.025 & $4.00 \cdot 10^{-4}$ & $3.91 \cdot 10^{-6}$ & $3.10 \cdot 10^{-8}$ \\
\hline
\end{tabular}

TABle 2. $H^{1}$ Convergence results

\begin{tabular}{llll}
\hline $\mathrm{h}$ & $\left\|u-u_{h}^{1}\right\|_{1}$ & $\left\|u-u_{h}^{2}\right\|_{1}$ & $\left\|u-u_{h}^{3}\right\|_{1}$ \\
\hline 0.2 & $7.92 \cdot 10^{-1}$ & $1.09 \cdot 10^{-1}$ & $1.10 \cdot 10^{-2}$ \\
0.1 & $3.72 \cdot 10^{-1}$ & $2.44 \cdot 10^{-2}$ & $1.08 \cdot 10^{-3}$ \\
0.05 & $1.83 \cdot 10^{-1}$ & $5.88 \cdot 10^{-3}$ & $1.25 \cdot 10^{-4}$ \\
0.025 & $8.93 \cdot 10^{-2}$ & $1.43 \cdot 10^{-3}$ & $1.49 \cdot 10^{-5}$ \\
\hline
\end{tabular}

Remarque 4.3. Note that the convergence results above clearly shows that our formulation checked the best convergence orders certified by the finite element theory. In addition, the above convergence results are obtained in the nonconforming configuration which illustrates the flexibility of the mortar element method. 


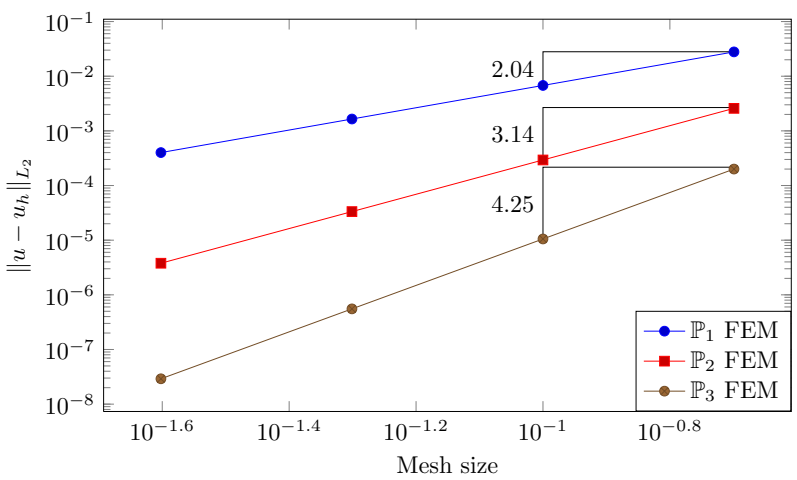

6.1. $L^{2}$ convergence

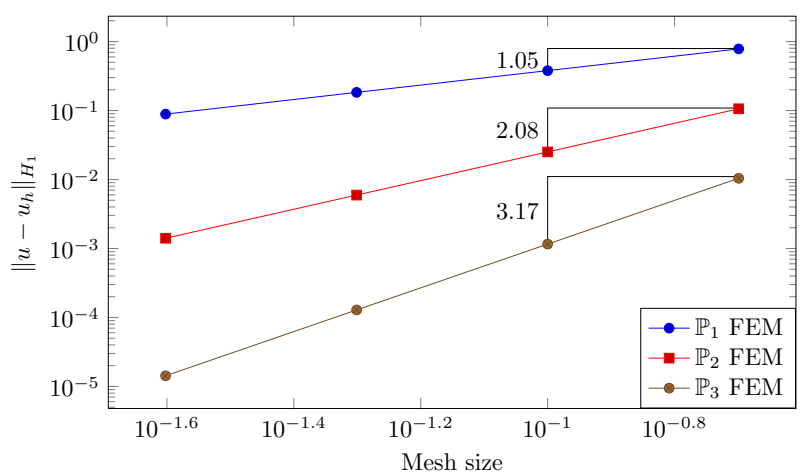

6.2. $H^{1}$ convergence

FiguRE 6. Convergence curves

\section{Conclusions}

This paper clearly shows that our parallel computational framework scales on the small scale architectures for solving the linear system arising from the mortar finite element method in 2D and 3D with the arbitrary number of subdomain partitions using the block-diagonal preconditioners. Our current work is focused on the implementation of the substructuring preconditioners $[9,10]$ for the discrete Steklov-Poincaré operator on interfaces so that the number of iterations is maintained as low as possible that is needed for a good scaling on very large scale architectures.

\section{ACKNOWLEDGEMENTS}

The authors would like to thank Vincent Chabannes for many fruitful discussions. Abdoulaye Samake and Christophe Prud'homme acknowledge the financial support of the project ANR HAMM ANR-2010-COSI-009.

This work was granted access to the HPC resources of TGCC@CEA made available within the Distributed European Computing Initiative by the PRACE-2IP, receiving funding from the European Community's Seventh Framework Programme (FP7/2007-2013) under grant agreement RI-283493 and of SuperMuc@LRZ available within the Distributed European Computing Initiative by the PRACE-3IP under grant agreement RI-312763.

\section{REFERENCES}

[1] Boost c++ libraries. http://www.boost.org.

[2] G.S. Abdoulaev, Y. Achdou, Y.A. Kuznetsov, and C. Prud'homme. On a parallel implementation of the mortar element method. RAIRO-M2AN Modelisation Math et Analyse Numerique-Mathem Modell Numerical Analysis, 33(2):245-260, 1999.

[3] Satish Balay, Kris Buschelman, Victor Eijkhout, William D. Gropp, Dinesh Kaushik, Matthew G. Knepley, Lois Curfman McInnes, Barry F. Smith, and Hong Zhang. PETSc users manual. Technical Report ANL-95/11 - Revision 2.1.5, Argonne National Laboratory, 2004.

[4] Satish Balay, Kris Buschelman, William D. Gropp, Dinesh Kaushik, Matthew G. Knepley, Lois Curfman McInnes, Barry F. Smith, and Hong Zhang. PETSc Web page, 2001. http://www.mcs.anl.gov/petsc.

[5] Satish Balay, Victor Eijkhout, William D. Gropp, Lois Curfman McInnes, and Barry F. Smith. Efficient management of parallelism in object oriented numerical software libraries. In E. Arge, A. M. Bruaset, and H. P. Langtangen, editors, Modern Software Tools in Scientific Computing, pages 163-202. Birkhäuser Press, 1997.

[6] F. Ben Belgacem and Y. Maday. The mortar element method for three-dimensional finite elements. R.A.I.R.O. Modél. Math. Anal., 31:289-302, 1997.

[7] M. Benzi, G.H. Golub, and J. Liesen. Numerical solution of saddle point problems. Acta numerica, 14(1):1-137, 2005.

[8] C. Bernardi, Y. Maday, and A. Patera. A new nonconforming approach to domain decomposition:the mortar element method. Nonlinear Partial Differential Equations and their Applications, 1993. 
[9] S. Bertoluzza and M. Pennacchio. Preconditioning the mortar method by substructuring: The high order case. Applied Numerical Analysis \& Computational Mathematics, 1(2):434-454, 2004.

[10] Silvia Bertoluzza and Micol Pennacchio. Analysis of substructuring preconditioners for mortar methods in an abstract framework. Applied Mathematics Letters, 20(2):131 - 137, 2007.

[11] Jie Chen, L.C. McInnes, and H. Zhang. Analysis and practical use of flexible BICGSTAB. Technical Report ANL/MCS-P30390912, Argonne National Laboratory, 2012.

[12] H.C. Elman, D.J. Silvester, and A.J. Wathen. Finite Elements and Fast Iterative Solvers:with Applications in Incompressible Fluid Dynamics: with Applications in Incompressible Fluid Dynamics. Numerical Mathematics and Scientific Computation. OUP Oxford, 2005.

[13] G.H. Golub and Q. Ye. Inexact preconditioned conjugate gradient method with inner-outer iteration. SIAM Journal on Scientific Computing, 21(4):1305-1320, 1999.

[14] Vicente Hernandez, Jose E. Roman, and Vicente Vidal. SLEPc: A scalable and flexible toolkit for the solution of eigenvalue problems. ACM Transactions on Mathematical Software, 31(3):351-362, 2005.

[15] M.F. Murphy, G.H. Golub, and A.J. Wathen. A note on preconditioning for indefinite linear systems. SIAM Journal on Scientific Computing, 21(6):1969-1972, 2000.

[16] Christophe Prud'homme. A domain specific embedded language in $\mathrm{C}++$ for automatic differentiation, projection, integration and variational formulations. Scientific Programming, 14(2):81-110, 2006.

[17] Christophe Prud'homme. Life: Overview of a unified C++ implementation of the finite and spectral element methods in 1d, 2d and 3d. In Workshop On State-Of-The-Art In Scientific And Parallel Computing, Lecture Notes in Computer Science, page 10. Springer-Verlag, 2007.

[18] Y. Saad. A flexible inner-outer preconditioned GMRES algorithm. SIAM Journal on Scientific Computing, 14(2):461-469, 1993.

[19] Y. Saad. Iterative methods for sparse linear systems. Society for Industrial and Applied Mathematics, 2003.

[20] Youcef Saad. A flexible inner-outer preconditioned gmres algorithm. SIAM J. Sci. Comput., 14(2):461-469, March 1993. 